\begin{tabular}{ccc}
\hline International Journal of Engineering \& Technology, 7 (4.19) (2018) 788-793 \\
International Journal of Engineering \& Technology \\
SPC & Website: www.sciencepubco.com/index.php/IJET \\
Research paper &
\end{tabular}

\title{
Enhanced Evolutionary Sequential Minimal Optimization Model for Inflation Prediction
}

\author{
Eman S. Al-Shamery*1, Hussein A. Al - Gashamy ${ }^{2}$ \\ IT College. Babylon University, Iraq, Department of Software, \\ Faculty of Information Technology, University of Babylon, Iraq \\ *Corresponding Author Email: emanalshamery@itnet.uobabylon.edu.iq
}

\begin{abstract}
The control of inflation rate is at the core of monetary policy making. Therefore, there is very great interest in reliable inflation forecasts by central bankers to help them achieve this aim. The aim of this investigation has been to forecast inflation in case of the United States as accurately as possible. This paper proposes a new forecasting model called Sequential Minimal Organization (SMOreg-3passes) for regression predictions. SMOreg-3passes consists of four steps, they are technical indicators generation, feature selection, normalization regression and regression forecaster. The proposed model evaluated using two regression measurements (Mean Absolute Error (MAE) and Root Mean Square Error (RMSE)). Our evidence from the SMOreg-3passes model suggests that the chronology of time series has great influence on future forecasting and the error in forecasting the past has an exponential impact on the current data. The results showed that the proposed model outperformed the traditional SMO and Multiple Layer Perception (MLP) methods.
\end{abstract}

Keywords: Inflation Rate, Technical Indicators, Feature Selection, Sequential Minimal Optimization (SMO).

\section{Introduction}

Inflation is one of the most important variables of economic models which measures the amount of change in the prices level of services and goods consumed by households that can affect the whole economy and government policy as it touches people's life and social stability. Consequently, it is very important to maintain inflation at threshold level and to maintain a rapid growth in the economy. High inflation with a momentum can be an indication to detrimental economic, political, and social effects, while low inflation may have a negative impact on the growth. Affected by several number of macroeconomic indicators such as: the federal funds rate, gross domestic product, commodity prices, consumer prices, consumption, investment, the GDP deflator, the producer price index, interest rates, stock prices, and the unemployment rate and others [Domenico et al., 2008]. Thus, the precise inflation forecasts are of the most importance for monetary policy and investment strategies[Iversen et al., 2016],specifically: in the calculation of real interest rates and setting it for targeting inflation. Forecasting is one of the main objectives of econometric modeling. Generally, there are two types of forecasting economic and statistical models. The first type is based on the historical data used for short term forecasting (univariate models), while the other type is based on multivariate models used for long term forecasting such as Vector Autoregressive model and cointegration. Philips curve analysis and random walk method were employed by Hubrichto forecast the inflation rate in the European region[Hubrich, 2005].ARIMA model was used to forecast Consumer Price Index that represents the main indicator of inflation rate [Dongdong, 2010].

A comparative study of the performance of VAR ,factor, and ARIMA models in forecasting the Australian inflation rate, produced results that showed the regressions favored factor model over Vector Autoregressive and ARIMA[Moser et al., 2007].according to another study[Marcellino, 2002], it was reported that nonlinear models perform better than linear and time-varying parameter models for predicting industrial production, inflation, and unemployment in the European region, Their study was extended that the real economic system is generally "nonlinear"[Barra et al., 2010].

This paper is organized as follows: the next section discusses some of the related work of using various machine learning and data mining techniques of crude oil prediction. In Section3, provides a quick overview of the concept of SVM's theory for regression, technical indicators, and Particle Swarm Optimization method. The experimental results are presented in Section 5 whereas the last section is the conclusion of this paper.

\section{Related Work}

In [Moser et al., 2007] the authors used the factor model as well as Auto Regressive Integrated Moving Average(ARIMA) and Vector Auto Regressive (VAR) to generate (12) month forecasts of Australian inflation and its Harmonized Index of Consumer Pricessub-indices. The results find out that the factor had the highest accuracy but the weakness lies in predicting the inflation rate, as it is not enough to use historical data; some external factors affect the inflation rate, like macroeconomic factors.

In the context of machine learning and artificial intelligence. Many studies have reported significant effects of ANN model for forecast Indian, Mexican and Chinese inflation, respectively, these studies used Back Propagation Neural Network, which considered that the exchange rate may have an impact on the inflation; it was thus revealed that ANNmodel is suitable for dealing with the 
nonlinear problem[Hurtado et al., 2013; Rudra, 2013; Wen et al., 2014]. In [Sari et al., 2016]the researchers employed the Back propagation Neural Network model to forecast the inflation in the future using a time series data of Consumer Price Index and inflation rate, additionally implemented the Sugeno Fuzzy Interference System model as a comparison method. The Inflation rate data in Indonesia from July-2005 to Dec.-2013 in which (20) records range from Mar.-2008 to Oct.-2009 for the testing data And the rest for training. Consumer Price Index that can affect the inflation rate is employed as external factors. The investigation shows that the proposed model has superior performance than the comparison method with error value reach 0.204 .

However, Neural Network implied a hazardous over fitting and required a large number of parameters, which represent a crucial issue for weak generalization[Eman and Ameer, 2018]. In recent years, the non-parametric Support Vector Regression (SVR) is more favorable for inflation predictability which combines the advantage of $\mathrm{NN}$ and the minimal structural risk principles. it has an excellent generalized performance for forecasting problems. The genetic algorithm was introduced to optimize the support vector machine [Guo et al., 2012] and the wavelet transform has been employed to remove the fluctuant components and ant colony optimization algorithm to optimize the parameters of SVM .The promising (ACO-SVM) established to predict share price in terms of predictive accuracy and generalization ability[Fang and Bai, 2009].

A new Hybrid Genetic Algorithm integrated with SVR model introduced by [Sermpinis et al., 2014]for forecasting United State unemployment and inflation. This model genetically optimizes the Support Vector Machine parameters to adapts to the optimal subset of features from a potential inputs feature space. The study thus thoroughly covers data over the period of Jan-1974 to Dec2012 compete with three traditional models used in technical analysis (Moving Average Convergence Divergence (MACD), Random Walk(RW),ARMA) and three popular computational intelligent methods (Recurrent Neural Network(RNN), Multiple Layer Perceptron(MLP), Genetic Programming (GP)).The result reveals that GA-SVR outperforms the other benchmarks with minimum accuracy (0.0049) and maximum( 0.0067) for MAE while $(0.0058-0.0072)$ for RMSE. In this research a modified Sequential Minimal Optimization was established for predicting inflation in the united states.

\section{Background}

\subsection{Inflation Forecasting Techniques}

\section{- Sequential Minimal Optimization (SMO)}

Due to large size in the objective function for the optimization problem in Support Vector Regression(SVR):

$\operatorname{Max} W(\alpha)=\sum_{i=1}^{n} \alpha_{i^{-}} \frac{1}{2} \sum_{i=1}^{n} \sum_{i=1}^{n} \alpha_{i} \alpha_{j} y_{i} y_{j} K\left(X_{i}, X_{j}\right)$

\section{Subject to:}

$\sum_{i=1}^{n} \alpha_{i} y_{i}=0$

$0 \leq \alpha_{i} \leq C \quad ; i=1,2, \ldots \ldots e$

Where $\mathrm{K}\left(\mathrm{X}_{\mathrm{i}}, \mathrm{X}_{\mathrm{j}}\right)$ represents the kernel function, the Quadratic problem that arises from SVRs cannot be solved efficiently by using standard numerical Quadratic Problem approaches especially for large size problem.

Different algorithms are proposed for solving the problem of dual function. Platt proposed a Sequential Minimal Optimization algorithm for classification problems, that iteratively selecting the working set of size two and optimize the objective function according to them by solving sub problem analytically[Platt, 1999].The process repeated iteratively until KKT conditions are satisfy for all training instance. Smola and Schölkopf extend the
SMO algorithm for solving the regression problems[Smola et al., 2004].

Four different cases must be distinguish

$\left(\dot{\alpha}_{\imath}, \dot{\alpha}_{\jmath}\right),\left(\boldsymbol{\alpha}_{i}, \dot{\alpha}_{j}\right),\left(\dot{\alpha}_{\imath}, \boldsymbol{\alpha}_{j}\right),\left(\boldsymbol{\alpha}_{i}, \boldsymbol{\alpha}_{j}\right)$ for regression problems, thus one obtain from the summation of the constrains:

$\left(\boldsymbol{\alpha}_{j}-\dot{\alpha}_{\boldsymbol{j}}\right)+\left(\boldsymbol{\alpha}_{\boldsymbol{i}}-\boldsymbol{\alpha}_{\boldsymbol{l}}\right)=\gamma$

Where $\boldsymbol{\alpha}_{\boldsymbol{j}} \in[0, \mathrm{Cj}], \boldsymbol{\alpha}_{\boldsymbol{i}} \in[1, \mathrm{~h}], 1$ and $\mathrm{h}$ are defined as in table 1:

Table 1: Boundary of the feasible region

\begin{tabular}{|c|l|l|}
\hline & \multicolumn{1}{|c|}{$\boldsymbol{\alpha}_{j}$} & \multicolumn{1}{c|}{$\dot{\alpha}_{\boldsymbol{J}}$} \\
\hline $\boldsymbol{\alpha}_{\boldsymbol{i}}$ & $\mathrm{l}=\operatorname{Max}\left(\mathrm{o}, \gamma-\mathrm{C}_{\mathrm{j}}\right)$ & $\mathrm{l}=\operatorname{Max}(\mathrm{o}, \gamma)$ \\
& $\mathrm{h}=\operatorname{Min}\left(\mathrm{C}_{\mathrm{i}}, \gamma\right)$ & $\mathrm{h}=\operatorname{Min}\left(\mathrm{C}_{\mathrm{i}}, \hat{C}_{J}+\gamma\right)$ \\
\hline$\dot{\alpha}_{\boldsymbol{\imath}}$ & $1=\operatorname{Max}(\mathrm{o},-\gamma)$ & $\mathrm{l}=\operatorname{Max}\left(0,-\gamma-\dot{C}_{J}\right)$ \\
& $\mathrm{h}=\operatorname{Min}\left(\dot{C}_{l},-\gamma+\mathrm{C}_{\mathrm{j}}\right)$ & $\mathrm{h}=\operatorname{Min}\left(\dot{C}_{l},-\gamma\right)$ \\
\hline
\end{tabular}

In this algorithm two threshold parameters value $b_{u p}$ and $b_{\text {low }}$ and checking optimality with the condition:

$\mathrm{B}_{\text {low }} \leq \mathrm{B}_{\text {up }}+2_{\varrho}$

SMO chooses two elements $\boldsymbol{\alpha}_{\boldsymbol{i}}$ and $\boldsymbol{\alpha}_{\mathrm{j}}$ to jointly optimize, then find the optimal values for those two parameters given that all the others are fixed, and updates the $\boldsymbol{\alpha}$ vector accordingly. A heuristic search is employed to select the two points through two separate heuristics: the first Lagrange multiplier provide the outer loop of the algorithm that iterates over the entire training one to determine whether each instance not satisfy the KKT conditions. If an instance violates these conditions, then it is qualified for optimization. After the first pass through the training set the outer loop iterates over all instance with non-bound Lagrange multipliers $\left(0<\alpha_{i}<C\right)$. This loop repeated over all the nonbound instances until all of them obey the KKT within $\varepsilon$. Then the outer loop returns and repeats on the entire training package. The outer loop keeps switching between one passé over the entire training set and multiple passes over the non-bound subset until the entire training set obeys the KKT conditions within $\varepsilon$. The optimization of multipliers is performed analytically. And the order in which data points are chosen for updating affects the rate of convergence of the algorithm. Heuristic measures such as the degree of violation of the KKT conditions can be used to ensure very effective convergence rates in practice. Most four basic kernel currently are using with SVM are shown in table2.

Table 2: kernel function

\begin{tabular}{|c|c|}
\hline Kernel function & Expression \\
\hline Linear & $\mathbf{x i}^{\mathbf{T}} \cdot \mathbf{x}_{\mathbf{j}}$ \\
\hline Polynomial & $\left(\mathbf{x i}^{\mathbf{T}} \cdot \mathbf{x}_{\mathbf{j}}+\boldsymbol{\theta}\right)^{\mathbf{r}}$ \\
\hline Radial Basis Function & $-\exp \frac{\left\|\mathbf{x}_{\mathbf{i}} \cdot \mathbf{x} \mathbf{j}\right\|^{2}}{\sigma^{2}}$ \\
\hline Sigmoid & $\tan \left(\mathbf{x i}^{\mathbf{T}} \cdot \mathbf{x}_{\mathbf{j}}+\boldsymbol{\theta}\right)$ \\
\hline
\end{tabular}

- Radial Basis Function(RBF) Kernel: RBF is type of feed forward network (FFNN), most commonly used with SVM due to its attractive characteristics for mapping input data into large dimensional space and a favorable kernel when there is little or no knowledge about the data distribution[Liu et al., 2012]. The mathematical equation for the RBF kernel is given by:

$K\left(X_{i}, X_{j}\right)=\exp \left(-\gamma\|X i-X j\|^{2}\right)$

$\gamma=\frac{1}{2 \sigma^{2}}, \gamma>0$

Where exp. is the exponent value and $\sigma$ represents the width of RBF kernel (degree of nonlinearity) with value range between [0 , $\infty$ ], if the value set to zero then learning algorithm susceptible to over fitting and to under fitting if $\sigma$ set to infinity, so that $\sigma$ represents the most crucial parameter for robust performance[Eman and Walaa,2018].

In this paper (RBF) kernel and Poly Kernel have been used for mapping input data to high dimensional .After using the optimal 
constraint $\mathrm{w}^{0}$ and $\mathrm{b}^{0}$ into Eq. 1 , then it will be in the following form:

$f(x)=\sum_{i=1}^{n}\left(\dot{\alpha}_{\boldsymbol{i}}^{\mathbf{0}}-\boldsymbol{\alpha}_{\boldsymbol{i}}^{\mathbf{0}}\right) \boldsymbol{K}(\boldsymbol{x}, \boldsymbol{x})+b$

- Multiple Layer Perceptron(MLP): MLP is a class of artificial neural network with hidden layer(one or more).The neurons represent the nodes of each layer with a nonlinear activation function (often used sigmoid function). MLP consists of layer of input, one or more hidden layer, and output layer of nodes interconnected in a feed forward direction. Fig.1provid an illustration of MLP.

MLP is a deep learning method that utilize back propagation(BP) as technique for training, BP consist of forward and backward phases, the first phase fed the inputs to the nodes of first hidden layer to perform the activities according to the activation function from input to output layer while the second phase exploits the error between actual and desired values from the output layer to propagate the input layer in order to modify the learning weights.

\subsection{Technical Indicators Generation}

This paper used (26) indicator, which can be categories into lagging and leading indicators that enhance the performance of proposed system. Mathematical formula and the periods are shown below.

Table 3: The period of TI's

\begin{tabular}{|c|c|c|c|c|}
\hline NO. & $T I s$ & Abbreviation & $\begin{array}{l}\text { Default } \\
\text { Period }\end{array}$ & $\begin{array}{c}\text { Suggested } \\
\text { Period }\end{array}$ \\
\hline 1 & AROON & Aroon & 6 & 7 \\
\hline 2 & AROON Oscillator & AroonOsc & 6 & 7 \\
\hline 3 & $\begin{array}{c}\text { Plus Directional } \\
\text { Movement }\end{array}$ & plus-DM & 12 & 14 \\
\hline 4 & $\begin{array}{c}\text { Minus Directional } \\
\text { Movement }\end{array}$ & minus-DM & 12 & 14 \\
\hline 5 & $\begin{array}{l}\text { Plus Directional } \\
\text { Indicator }\end{array}$ & plus-DI & 12 & 14 \\
\hline 6 & $\begin{array}{l}\text { Minus Directional } \\
\text { Indicator }\end{array}$ & minus $-d i$ & 12 & 14 \\
\hline 7 & Standard Deviation & St.Dev. & 6 & 5 \\
\hline 8 & Variance & $V A R$ & 6 & 5 \\
\hline 9 & Linear Regression & $\begin{array}{c}L R- \\
\text { Linearage }\end{array}$ & 12 & 14 \\
\hline 10 & $\begin{array}{l}\text { Linear Regression- } \\
\text { Intercept }\end{array}$ & $L R$-intercept & 12 & 14 \\
\hline 11 & Linear Regression-Slope & LR-slope & 12 & 14 \\
\hline 12 & Linear Regression-Angle & LR-Angle & 12 & 14 \\
\hline 13 & Absolute Percentage & $A P O$ & 12 & 14 \\
\hline 14 & Williams & $\% R$ & 6 & 7 \\
\hline 15 & $\begin{array}{c}\text { Kaufman Moving } \\
\text { Average }\end{array}$ & Kama & 10) & 14 \\
\hline 16 & Momentum & $M O M$ & 6 & 7 \\
\hline 17 & $\begin{array}{l}\text { Commodity Channel } \\
\text { Index }\end{array}$ & $c c i$ & 6 & 14 \\
\hline 18 & Relative Strength Index & $R S I$ & 12 & 14 \\
\hline 19 & Average True Range & $A T R$ & 6 & 7 \\
\hline 20 & Mid-Point & Mid & 6 & 7 \\
\hline 21 & $\begin{array}{c}\text { Moving Average } \\
\text { Convergence Divergence }\end{array}$ & $M A C D$ & 35 & 35 \\
\hline 22 & $\begin{array}{c}\text { Exponential Moving } \\
\text { Average }\end{array}$ & $E M A$ & 30 & 30 \\
\hline 23 & Simple Moving Average & $S M A$ & 30 & 30 \\
\hline 24 & $\begin{array}{l}\text { Average Directional } \\
\text { Index }\end{array}$ & $A D X$ & 23 & 14 \\
\hline 25 & minimum & Low & 5 & 30 \\
\hline 26 & Maximum & High & 5 & 30 \\
\hline
\end{tabular}

\subsection{Feature Selection Technique}

- Particle Swarm Optimization(PSO): It isan evolutionary computation(EC) technique introduced by [Kennedy and Eberhart, 1995]. PSO inspired by social behavior likea school of fish and birds flocking. Compared with other EC algorithms such as GA's, it can converge more quickly[Tran et al., 2014]. Each solution is called a particle without any weight and volume. In this paper, PSO is employed as FS approach to enhance the forecasting regression performance, minimizing the number of features and reducing the computational time. Experiments on inflation dataset show that PSO can feature subset with minimum features and higher prediction performance than using all features.

vector $f i=\left(f_{i 1}, f_{i 2}, \ldots, f_{i D}\right)$ presenting the position of particle $i$, vector $V i=\left(V_{i 1}, V_{i 2}, \ldots, V_{i D}\right)$ represent the velocity of the $i$ th particle, For any particle, The best previous position is recorded as the personal best ( PBest) whereas the global best(GBest) represents the best solution discovered by the whole swarm. According to the GBest and PBest, a population of random solutions has been initialized for the swarm. The algorithm updating the positions of particles and velocities searches for the best solution y using the following formulae:

$V_{i d}{ }^{t+1}=w^{*} V_{i d}{ }^{t}+C 1 * R 1 *\left(P_{i d^{-}} f_{i d}{ }^{t}\right)+C 2 * R 2 *\left(P_{g d}-f_{i d}{ }^{t}\right)$

$f_{i d}^{t+1}=f_{i d}^{t}+V_{i d}{ }^{t+1}$

Where (t) represent the iteration, $\mathrm{C} 1$ and $\mathrm{C} 2$ are constant accelerations, $R 1$ and $R 2$ represents random values distributed uniformly between $[0,1], P_{i d}$ means the $P_{B e s t}$ while $P_{g d}$ presents the $G_{B e s t}$, and W represents the weight that makes the balance between local and global search. The velocity $V_{i d}{ }^{t+1} \in\left[-V_{M A X}, V_{M A X}\right]$ where the value of $\mathrm{V}_{\text {MAX }}$ based on the problem where suggested to $10-20 \%$ of the dynamic range of the variable in each dimension. this paper employed (Geometric PSO ) algorithm where velocity does not exist, instead of that mutation is present and the parameters $w 1, w 2$, and $w 3$ are positive. The equation of position update is the convex combination as computed by the formula below[Moraglio et al., 2007]:

$y i=C x((f i, w 1),(g, w 2),(x, w 3))$

- CFS Subset Evaluation: CFS is a filter algorithm that give ranks for feature subsets according to a correlation with the class based heuristic evaluation function(HEF), which is proposed by[Hall and Smith, 1999].The HEF tends to find subsets of features that are strongly correlated with the class.

Redundant features can be strongly correlated with one or more features so they should be screened out. While Irrelevant features have a poor correlation with the class so that they should be ignored. The acceptance of a feature will based on (9)which represents the core of CFS which assign a ranking for the feature.

$M_{I}=\frac{N \overline{r_{f c}}}{\sqrt{N+N(N+1) \overline{r_{f f}}}}$

Where $\left(\mathrm{M}_{\mathrm{I}}\right)$ represents the significant degree(merit) of a feature subset(I) containing $\mathrm{N}$ features, $\left(r_{f f}\right)$ is the mean inter-correlation between feature-feature. And $\left(r_{f c}\right)$ is the average correlation between class - feature.

\section{Proposed Model}

The architecture of the proposed model for inflation rate forecasting are illustrated in Fig.2.

\subsection{Dataset}

All the monthly data come from Energy Information Administration (EIA) of United States, which start from Jan1982 to Jun- 2017. In total (374)instances divided into two datasets: primary and future dataset, the primary data are divided into training and testing group, In total $(306,34)$ of instances respectively. The last three years (34) instance are treated as the future dataset, which will not be involved in the primary dataset. 


\subsection{Pre-processing Stage}

This stage consists of two steps include features extraction and transforming nominal to numeric.

- Feature Extraction: Extract standard features from raw inflation monthly rate (date, inflation Rate, high, low) are taken as inputs to proposed system. Fig. 3 shows the standard features.

- Nominal To Numeric: One step of preprocessing data to make it more suitable for the specified algorithm by changing the type of non-numeric attributes to a numeric type by using concatenation transformation function .

\subsection{Indicator Selection (IS)}

The objective of FS is to reduce the dimensionality of features space by removing irrelevant features and indicators and increasing the forecasting accuracy level. This paper employed CFS Selection algorithm as an evaluator for PSO method. In this method, only those subset of features(standard features and TI's) are selected which are highly correlated with the class and having low inter-correlation values. Geometric PSO algorithmconsists of many steps as shown in algorithm (1).10-foldcross-validation used for the experimental results of CFS subset Evaluation listed in table3.

Table 4: The rank of features

\begin{tabular}{|c|c|l|c|c|l|}
\hline $\begin{array}{c}\text { No } \\
\boldsymbol{n}\end{array}$ & $\begin{array}{c}\text { number of folds } \\
(\mathbf{\%})\end{array}$ & Attribute & $\begin{array}{c}\text { No } \\
\boldsymbol{1}\end{array}$ & $\begin{array}{c}\text { numbe } \\
\mathbf{r} \text { of } \\
\mathbf{f o l d s} \\
\mathbf{( \% )}\end{array}$ & $\begin{array}{l}\text { Attribut } \\
\mathbf{e}\end{array}$ \\
\hline $\mathbf{1}$ & 2 & Max & 15 & 0 & Lr-angle \\
\hline $\mathbf{2}$ & 10 & Min & 16 & 0 & $\begin{array}{l}\text { Lr- } \\
\text { intercept }\end{array}$ \\
\hline $\mathbf{3}$ & 8 & Midpoint & 17 & 0 & $\begin{array}{l}\text { Minus- } \\
\text { DM }\end{array}$ \\
\hline $\mathbf{4}$ & 1 & MACD & 18 & 2 & $\begin{array}{l}\text { LR- } \\
\text { lineage }\end{array}$ \\
\hline $\mathbf{5}$ & 0 & SMA & 19 & 10 & APO \\
\hline $\mathbf{6}$ & 0 & EMA & 20 & 0 & KAMA \\
\hline $\mathbf{7}$ & 0 & ADX & 21 & 0 & Var \\
\hline $\mathbf{8}$ & 0 & St.Dev. & 22 & 0 & ATR \\
\hline $\mathbf{9}$ & 10 & MOM. & 23 & 0 & Date \\
\hline $\mathbf{1 0}$ & 0 & RSI & 24 & 0 & CCI \\
\hline $\mathbf{1 1}$ & 1 & Plus-di & 25 & 0 & willer \\
\hline $\mathbf{1 2}$ & 10 & PLUS- & 26 & 0 & Aroon \\
\hline $\mathbf{1 3}$ & 2 & DM & & & $\begin{array}{l}\text { Aroon } \\
\text { Or-Slop }\end{array}$ \\
\hline $\mathbf{1 4}$ & 2 & Minus-di & & 0 & \\
\hline & & & & & \\
\hline
\end{tabular}

\subsection{Modified SMOreg-3passes}

In this stage, apropos method of SVR has been applied with respect to time series that split dataset into Q-segments, from those K segments(segment(1), segment(2), .., segment(k)) as the primary dataset while the future is(segment $(\mathrm{Q}))$. In the first pass, the primary dataset has been trained and tested with SMO reg and RBF-kernel. The predicted values and errors from the first pass for (K-1)segments will serve as training dataset for the last output segment $(\mathrm{k})$ in the second pass, the purpose of this procedure was to give more importance for recent months which act as the current period which in turn have more impacts on the future. Future dataset has been passed as same as pass one of primary dataset, the third passes exploit the current dataset from primary as training and the result of future as test to improve the accuracy of predicted future output with different Polynomial-Kernel. Fig.(4) shows the architecture of the proposed system.

\subsection{Forecasting Evaluation}

In this study, two measures RMSE and MAE are employed as indicators of the performance to compare the results obtained by the proposed model with those obtained from another competitive models.

$\mathrm{RMSE}=\frac{1}{k} \sqrt{\sum_{i=1}^{k} \mid} X_{i}-\left.X_{j}\right|^{2}$

$\mathrm{MAE}=\frac{1}{k} \sum_{i=1}^{k}\left|X_{i}-X_{j}\right|$

Where $X_{i}$ acts the actual value; $X_{j}$ represents the forecasting value; $K$ represents the test sample size.

\section{Experimental Results}

Dataset split into primary and future data set with respect to time series to exploit error in ordered form as shown in table 5.

Table 5: Dataset split for each pass

\begin{tabular}{|l|l|l|l|l|}
\hline \multirow{2}{*}{ passes } & Primary & future \\
\cline { 2 - 5 } & train & test & train & test \\
\hline Pass1 & $\mathbf{3 4 0}$ & $\mathbf{3 4 0}$ & $\mathbf{3 4}$ & $\mathbf{3 4}$ \\
\hline Pass2 & $\mathbf{3 0 6}$ & $\mathbf{3 4}$ & & \\
\hline Pass3 & $\mathbf{3 4}$ & $\mathbf{3 4}$ & \\
\hline
\end{tabular}

Two metrics (RMSE and MAE) are used in this study to evaluate the proposed regression predictor model(SMOreg-3passes). RBF kernel is utilized in the first pass of the Primary and Future dataset based on 10-fold cross-validation. MAE and RMSE for primary and future datasets are listed in tables 6 and 7 , respectively.

Table 6: MAE \& MASE for Primary dataset (pass1)

\begin{tabular}{|c|c|c|c|}
\hline Segment & Time(s) & MAE & RMSE \\
\hline $\mathbf{1}$ & 0.03 & 0.0081 & 0.0092 \\
\hline $\mathbf{2}$ & 0.03 & 1.3017 & 2.4103 \\
\hline $\mathbf{3}$ & 0.01 & 0.9636 & 1.278 \\
\hline $\mathbf{4}$ & 0.01 & 0.0042 & 0.0049 \\
\hline $\mathbf{5}$ & 0.02 & 0.0038 & 0.0045 \\
\hline $\mathbf{6}$ & 0.03 & 0.009 & 0.0114 \\
\hline $\mathbf{7}$ & 0.01 & 0.003 & 0.0036 \\
\hline $\mathbf{8}$ & 0.01 & 0.0041 & 0.0047 \\
\hline $\mathbf{9}$ & 0.02 & 0.0066 & 0.0085 \\
\hline $\mathbf{1 0}$ & 0.02 & 0.0075 & 0.0088 \\
\hline Avg. & 0.19 & 0.23116 & 0.37439 \\
\hline
\end{tabular}

Table 7: MAE \& MASE for future dataset (pass1)

\begin{tabular}{|c|c|c|c|}
\hline Segment & Time(s) & Mae & RMSE \\
\hline $\mathbf{1}$ & 0.0002 & 0.0027 & 0.0033 \\
\hline $\mathbf{2}$ & 0.0002 & 0.0021 & 0.0025 \\
\hline $\mathbf{3}$ & 0.0002 & 0.0048 & 0.0049 \\
\hline $\mathbf{4}$ & 0.0002 & 0.0016 & 0.0022 \\
\hline $\mathbf{5}$ & 0.02 & 0.0035 & 0.0039 \\
\hline $\mathbf{6}$ & 0.0002 & 0.0046 & 0.0046 \\
\hline $\mathbf{7}$ & 0.02 & 0.0005 & 0.0006 \\
\hline $\mathbf{8}$ & 0.0002 & 0.0018 & 0.0021 \\
\hline $\mathbf{9}$ & 0.0002 & 0.0016 & 0.0024 \\
\hline $\mathbf{1 0}$ & 0.0002 & 0.0012 & 0.0016 \\
\hline Avg. & 0.041 & 0.00244 & 0.00281 \\
\hline
\end{tabular}

In the second pass, our findings of RMSE and MAE with similar parameters and kernel of SMO reg algorithm for the last segment in the primary dataset (k) are shown in Table 8.On the other hand, different kernel function(Poly kernel) conjunct with the predictor are used for the third pass.

Table 8: MAE \& MASE for Primary dataset (pass2)
\begin{tabular}{|l|l|l|l|}
\hline Segment & Time & MAE & RMSE \\
\hline Segment $(\boldsymbol{k})$ & 0.03 & $\mathbf{0 . 0 0 8 4}$ & $\mathbf{0 . 0 1 0 1}$ \\
\hline
\end{tabular}


Additionally, a comparative study with two standard regression predictors MLP regression and traditional SMO applied on the same data set testing based cross-validation (10-fold).

Table 9: MAE \& MASE for different algorithm

\begin{tabular}{|l|c|c|c|c|}
\hline Algorithm & $\begin{array}{c}\text { Time- } \\
\text { training }\end{array}$ & Time-testing & MAE & RMSE \\
\hline SMO & 0.07 & 0.02 & 0.0095 & 0.0109 \\
\hline $\begin{array}{l}\text { MLP- } \\
\text { regression }\end{array}$ & 0.39 & 0.01 & 0.0077 & 0.0078 \\
\hline $\begin{array}{l}\text { SMOreg- } \\
\text { 3passes }\end{array}$ & 0.02 & 0.01 & 0.0003 & 0.0005 \\
\hline
\end{tabular}

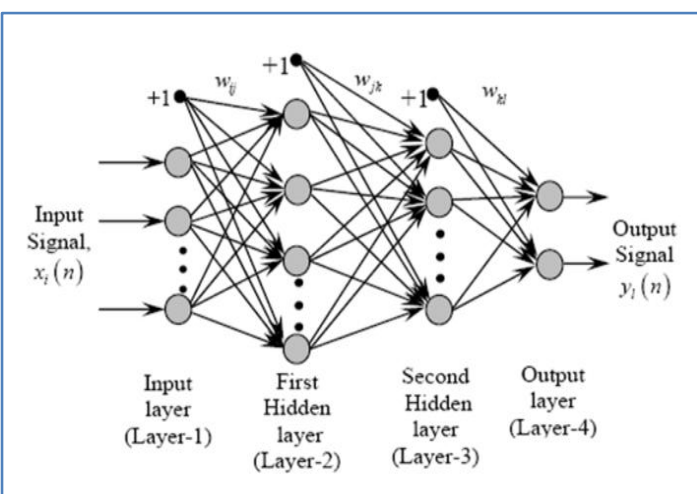

Fig. 1: Illustration of Multilayer Perceptron Network [Nimaje and Tripathy, 2015]

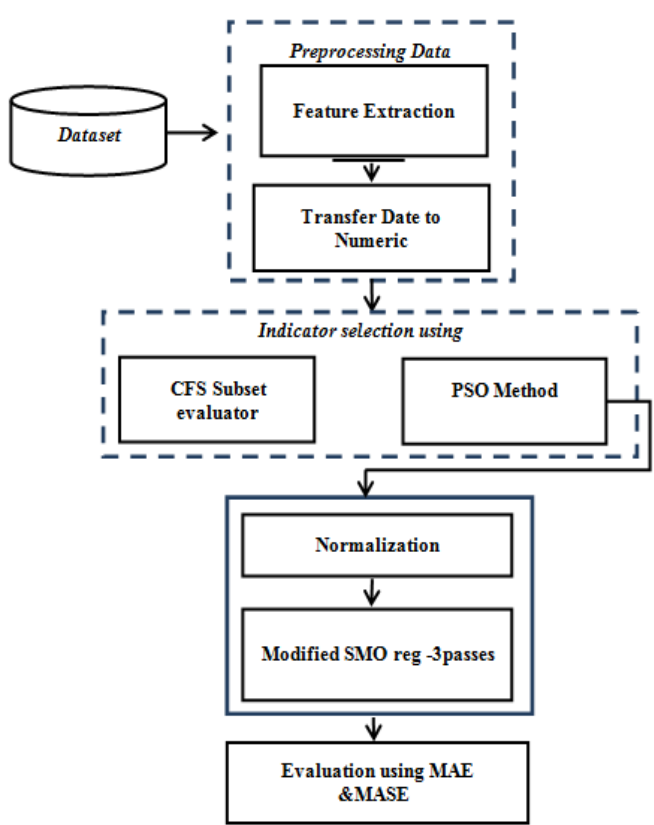

Fig. 2: The proposed system

\begin{tabular}{|c|c|c|c|}
\hline Date & Inflation Rate & Low & High \\
\hline $\mathbf{1 1 / 1 / 2 0 1 4}$ & 0.0132 & 0.0132 & 0.0207 \\
\hline $\mathbf{1 2 / 1 / 2 0 1 4}$ & 0.0076 & 0.0076 & 0.0199 \\
\hline $\mathbf{1 / 1 / 2 0 1 5}$ & -0.0009 & -0.0009 & 0.017 \\
\hline $\mathbf{2 / 1 / 2 0 1 5}$ & -0.0003 & -0.0009 & 0.0166 \\
\hline $\mathbf{3 / 1 / 2 0 1 5}$ & -0.0007 & -0.0009 & 0.0166 \\
\hline
\end{tabular}

Fig. 3: Feature Extraction

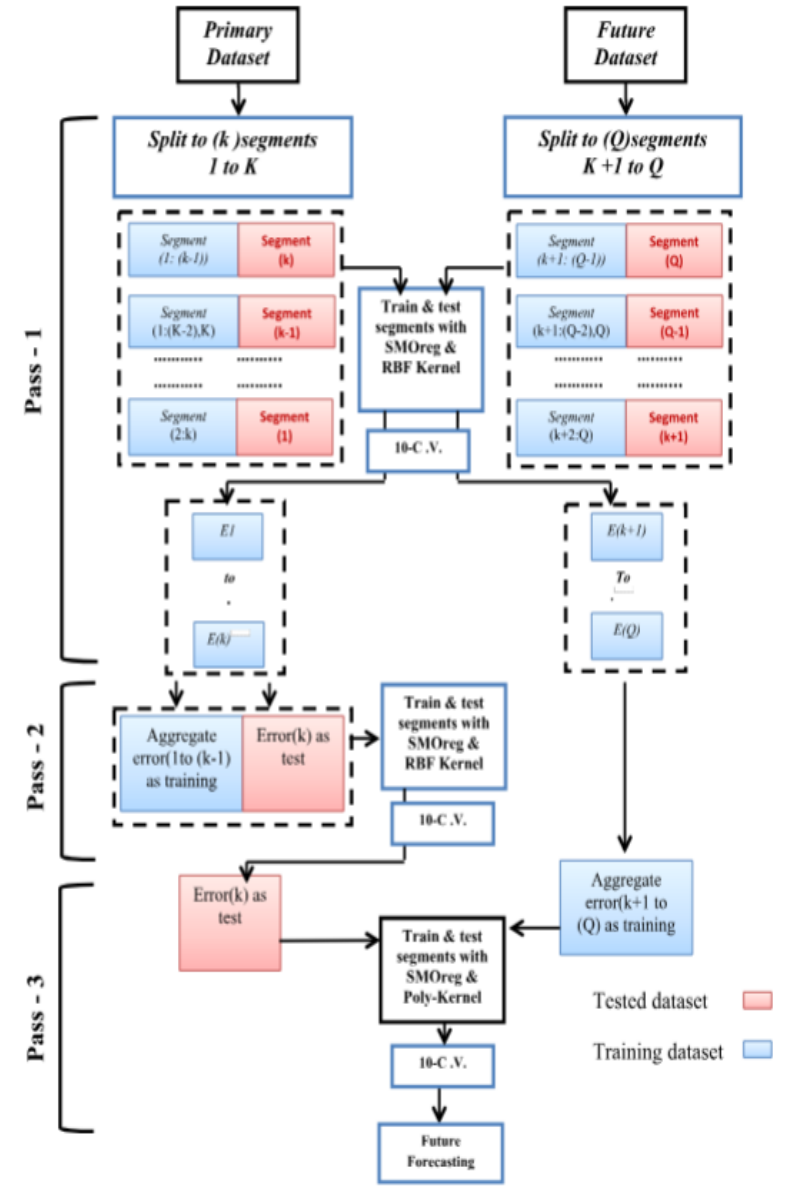

Fig. 4: The architectural of Modified SMOreg-3passes

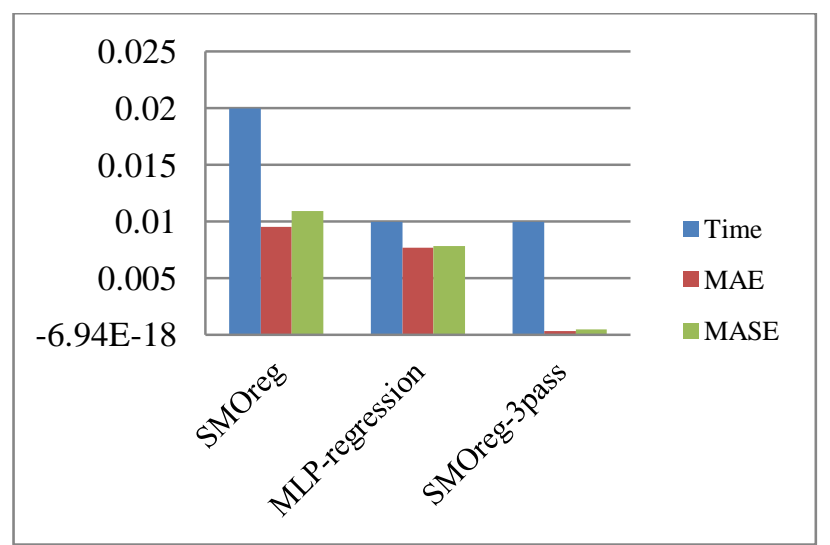

Fig. 5: MAE\&RMSE for future with different algorithms

Algorithm (1): Geometric PSO algorithm

Input: array of training instance consist of a vector of attribute values $\mathrm{f}_{\mathrm{i}}, \mathrm{z}=$
$1, \ldots, \mathrm{a}$, labeled with the target value $\mathrm{y}$.
Output: best subset of features
Begin
Step1. For $\mathrm{z}=1$ to $\mathrm{v}$ :
2.1 initialize position $\left(\mathrm{x}_{\mathrm{z}}\right)$ in the search space randomly.
end
step2. while stopping criteria not happen do
2.1 for all $\mathrm{z}$ do
2.2 set PBest $\mathrm{x}_{\mathrm{z}}$ as best position found so far by the particle
2.3 set GBest $\mathrm{g}$ as best position found so far by the whole swarm
end
step3. for all particle $i$ do
3.1 update position $f i$ using a randomized convex combination
$3.2 \mathrm{x}_{z}=\mathrm{Cx}\left(\left(\mathrm{f}_{z}, \mathrm{w} 1\right),(\mathrm{g}, \mathrm{w} 2),\left(\mathrm{x}_{\mathrm{z}}, \mathrm{w} 3\right)\right)$
3.3 perform mutate of $\mathrm{x}_{z}$
end




\section{Conclusions}

In summary, this paper presents a modified SMOreg-3passes model in economic forecasting and indicators selection. The proposed model is applied to US inflation with respect to time series. In addition, technical indicators have a great impact on the efficiency of forecasting model. Therefore, a special indicator selection method (PSO with CFS evaluator) has been applied to those indicators to select the best of them. The study revealed that PSO can produce good features selection by giving the estimation of the merit of attributes (11 of 29 features). Experimental results showed that the proposed methodology is a very promising SMO reg model for forecasting inflation rate with MAE and MASE better than all comparative models as shown in previous section.

\section{References}

[1] Barra, A., G. Genovese, and F. Guerra (2010), The Replica Symmetric Approximation of the Analogical Neural Network, Journal of Statistical Physics, 140(4), 784-796, doi: 10.1007/s10955-010-0020-y.

[2] Domenico, G., R. Lucrezia, and S. David (2008), Nowcasting: the real time informational content of macroeconomic data releasesRep., ULB -- Universite Libre de Bruxelles.

[3] Dongdong, W. (2010), The Consumer Price Index Forecast Based on ARIMA Model, paper presented at 2010 WASE International Conference on Information Engineering.

[4] Fang, X., and T. Bai (2009), Share Price Prediction Using Wavelet Transform and Ant Colony Algorithm for Parameters Optimization in SVM, paper presented at 2009 WRI Global Congress on Intelligent Systems.

[5] Guo, X., D. Li, and A. Zhang (2012), Improved Support Vector Machine Oil Price Forecast Model Based on Genetic Algorithm Optimization Parameters, AASRI Procedia, 1, 525-530, doi: https://doi.org/10.1016/j.aasri.2012.06.082

[6] Hall, M. A., and L. A. Smith (1999), Feature Selection for Machine Learning: Comparing a Correlation-Based Filter Approach to the Wrapper, in Proceedings of the Twelfth International Florida Artificial Intelligence Research Society Conference, edited, pp. 235-239, AAAI Press.

[7] Hubrich, K. (2005), Forecasting euro area inflation: Does aggregating forecasts by HICP component improve forecast accuracy?, International Journal of Forecasting, 21(1), 119-136, doi: https://doi.org/10.1016/j.ijforecast.2004.04.005.

[8] Hurtado, C., J. Luis, C. Fregoso, and J. Héctor (2013), Forecasting Mexican inflation using neural networks, paper presented at CONIELECOMP 2013, 23rd International Conference on Electronics, Communications and Computing.

[9] Iversen, J., S. Laséen, H. Lundvall, and U. S?derstr?m (2016), Real-Time Forecasting for Monetary Policy Analysis: The Case of Sveriges RiksbankRep., Sveriges Riksbank (Central Bank of Sweden).

[10] Kennedy, J., and R. Eberhart (1995), Particle swarm optimization, paper presented at Neural Networks, 1995. Proceedings., IEEE International Conference on.

[11] Liu, Z., M. J. Zuo, and H. Xu (2012), Parameter selection for Gaussian radial basis function in support vector machine classification, paper presented at 2012 International Conference on Quality, Reliability, Risk, Maintenance, and Safety Engineering.

[12] Marcellino, M. (2002), Instability and Non-Linearity in the EMURep., C.E.P.R. Discussion Papers.

[13] Moraglio, A., C. D. Chio, and R. Poli (2007), Geometric particle swarm optimisation, in Proceedings of the 10th European conference on Genetic programming, edited, pp. 125-136, SpringerVerlag, Valencia, Spain.

[14] Moser, G., F. Rumler, and J. Scharler (2007), Forecasting Austrian inflation, Economic Modelling, 24(3), 470-480, doi https://doi.org/10.1016/j.econmod.2006.10.003

[15] Nimaje, D. S., and D. P. Tripathy (2015), Assessment of Fire Risk of Indian Coals Using Artificial Neural Network Techniques, American Journal of Mining and Metallurgy, 3(2), 43-53, doi: 10.12691/ajmm-3-2-2.

[16] Platt, J. C. (1999), Fast training of support vector machines using sequential minimal optimization, in Advances in kernel methods, edited by S. Bernhard, lkopf, J. C. B. Christopher and J. S. Alexander, pp. 185-208, MIT Press

[17] Rudra, P. P. (2013), Forecasting Inflation in India: An Application of ANN Model, in Business, Technology, and Knowledge Management in Asia: Trends and Innovations, edited by P. Patricia Ordóñez de, pp. 32-42, IGI Global, Hershey, PA, USA.

[18] Sari, N., W. Mahmudy, and A. Wibawa (2016), Backpropagation on neural network method for inflation rate forecasting in Indonesia, 69-87 pp.

[19] Sermpinis, G., C. Stasinakis, K. Theofilatos, and A Karathanasopoulos (2014), Inflation and unemployment forecasting with genetic support vector regression, Journal of Forecasting, 33(6), 471-487, doi: 10.1002/for.2296.

[20] Smola, A. J., B. Sch, \#246, and lkopf (2004), A tutorial on support vector regression, Statistics and Computing, 14(3), 199-222, doi: 10.1023/b:stco.0000035301.49549.88.

[21] Tran, B., B. Xue, and M. Zhang (2014), Overview of Particle Swarm Optimisation for Feature Selection in Classification, Springer International Publishing, Cham.

[22] Wen, Y. C., L. N. Tan, and H. L. Wu (2014), Inflation Forecast Based on BP Neural Network Model, paper presented at Advanced Materials Research, Trans Tech Publ. 